\title{
Modelling the structure and kinematics of the Firework nebula: The nature of the GK Persei nova shell and its jet-like feature
}

\author{
E. Harvey ${ }^{1}$, M. P. Redman ${ }^{1}$, P. Boumis ${ }^{2}$, and S. Akras ${ }^{3}$ \\ 1 Centre for Astronomy, School of Physics, National University of Ireland Galway, University Road, Galway, Ireland \\ e-mail: [e.harvey2; matt.redman] @nuigalway.ie \\ 2 National Observatory of Athens, IAASARS, I. Metaxa \& V. Pavlou, Penteli, 15236 Athens, Greece \\ 3 Observatório do Valongo, Universidade Federal do Rio de Janeiro, Ladeira Pedro Antonio 43 20080-090 Rio de Janeiro, Brazil
}

Received 14 January 2016 / Accepted 29 August 2016

\begin{abstract}
Aims. The shaping mechanisms of old nova remnants are probes for several important and unexplained processes, such as dust formation and the structure of evolved star nebulae. To gain a more complete understanding of the dynamics of the GK Per (1901) remnant, an examination of symmetry of the nova shell is explored, followed by a kinematical analysis of the previously detected jet-like feature in the context of the surrounding fossil planetary nebula.

Methods. Faint-object high-resolution echelle spectroscopic observations and imaging were undertaken covering the knots which comprise the nova shell and the surrounding nebulosity. New imaging from the Aristarchos telescope in Greece and long-slit spectra from the Manchester Echelle Spectrometer instrument at the San Pedro Mártir observatory in Mexico were obtained, supplemented with archival observations from several other optical telescopes. Position-velocity arrays are produced of the shell, and also individual knots, and are then used for morpho-kinematic modelling with the SHAPE code. The overall structure of the old knotty nova shell of GK Per and the planetary nebula in which it is embedded is then analysed.

Results. Evidence is found for the interaction of knots with each other and with a wind component, most likely the periodic fast wind emanating from the central binary system. We find that a cylindrical shell with a lower velocity polar structure gives the best model fit to the spectroscopy and imaging. We show in this work that the previously seen jet-like feature is of low velocity.

Conclusions. The individual knots have irregular tail shapes; we propose here that they emanate from episodic winds from ongoing dwarf nova outbursts by the central system. The nova shell is cylindrical, not spherical, and the symmetry axis relates to the inclination of the central binary system. Furthermore, the cylinder axis is aligned with the long axis of the bipolar planetary nebula in which it is embedded. Thus, the central binary system is responsible for the bipolarity of the planetary nebula and the cylindrical nova shell. The gradual planetary nebula ejecta versus sudden nova ejecta is the reason for the different degrees of bipolarity. We propose that the "jet" feature is an illuminated lobe of the fossil planetary nebula that surrounds the nova shell.
\end{abstract}

Key words. methods: data analysis - methods: observational - astronomical databases: miscellaneous - stars: dwarf novae novae, cataclysmic variables - stars: jets

\section{Introduction}

With a proximity of $470 \mathrm{pc}$ (McLaughlin 1960; Harrison et al. 2013), GK Per (1901) is a nearby, historic, and spectacular postnova source. As the nearest and brightest of only two classical nova remnants observed within a planetary nebula to date, the other being V458 Vul (Wesson et al. 2008; Roy et al. 2012), it offers the best chance to study the evolution of nova explosion debris within a planetary nebula and thus potentially aids the understanding of both types of object.

A classical nova event is the result of thermonuclear runaway on the surface of a white dwarf accreting from, typically, a main sequence or a late G- or K-type star (Warner 1995). The accreted shell is ejected, at velocities ranging from $5 \times 10^{2}$ to typically $<5 \times 10^{3} \mathrm{~km} \mathrm{~s}^{-1}$ (Bode \& Evans 2008), once a critical pressure is reached at the core-envelope interface. Before ejection, mixing occurs between the accreted envelope and the white dwarf core through convection, leading to a heavyelement enrichment of the roughly solar composition envelope (Casanova et al. 2011). Dwarf novae, which are also exhibited by the GK Per system, result from an instability in the accretion disk surrounding the white dwarf star; the instability is caused by a disturbance in the magnetic field of the white dwarf leading to a brightening of 2-6 mag (Osaki 1996). These events accelerate winds to the order of $1-6 \times 10^{3} \mathrm{~km} \mathrm{~s}^{-1}$ (Cordova \& Mason 1982; Mauche \& Raymond 1987; Kafka \& Honeycutt 2004), generally faster than classical nova winds. Dwarf novae are important in terms of accretion disk physics as during their rise and fall the majority of the emission is from the disk (Osaki 1996).

The onset and progression of thermonuclear runaway in the surface envelope of post-enriched accreted hydrogen (and/or helium) is very difficult to explain (Casanova et al. 2011). However, detailed observations of the outflow allow for estimates of the total mass and abundances of the heavy elements ejected, as is explored in Helton et al. (2011). This provides constraints on the mechanism and efficiency of dredge-up from the underlying white dwarf. A full understanding of this process would greatly improve inputs into models for nuclear reactions that follow the thermonuclear runaway, which would improve the assessment of ejecta masses and composition (Casanova et al. 2011).

As noted by Ederoclite (2005), and previously by others (e.g. Ringwald et al. 1996, and references therein), that old novae are often neglected after their explosive lightcurves have reached quiescence. An important possible consequence of these systems 


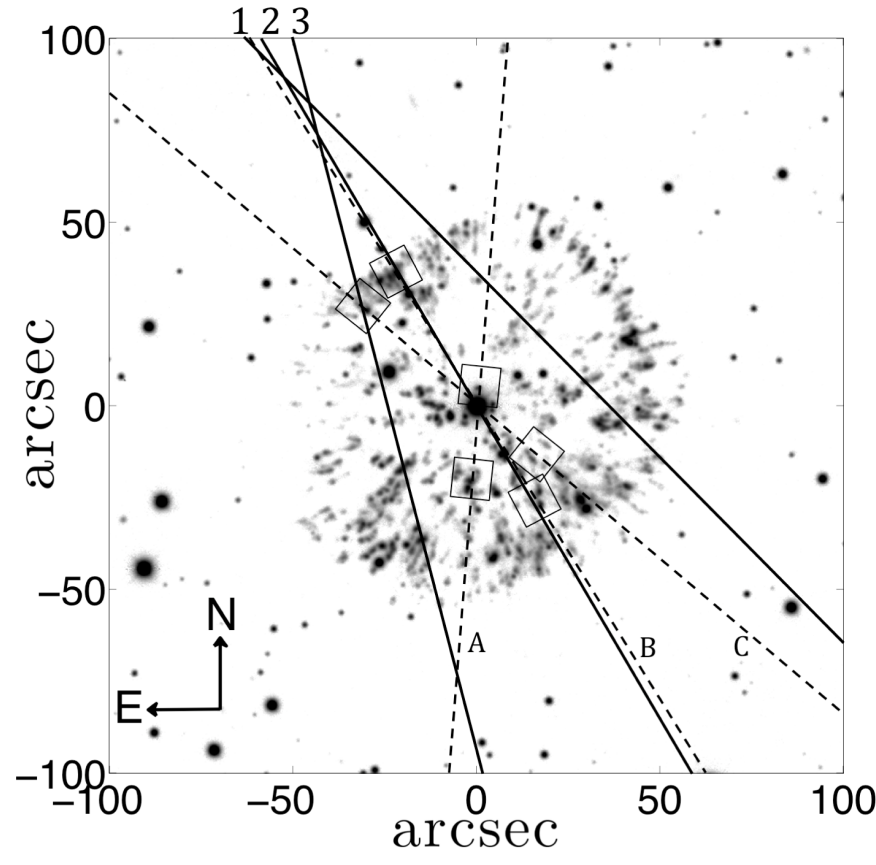

Fig. 1. Positions of the slits from MES-SPM overlaid on the stacked $\mathrm{H} \alpha$ Mayall image first presented in Shara et al. (2012). The slit marked 1 was placed at a PA of $45^{\circ}$, slit 2 at $30^{\circ}$ and slit 3 at $9^{\circ}$. All three slit positions cover the jet-like feature to the NE of the nova remnant as well as sections of the nova shell. Slit $1 \& 2$ observations were obtained on 2014-Nov-28 and slit 3 on 2015-Mar-27/28, see Table 2a. Slits marked $\mathrm{A}, \mathrm{B}$, and $\mathrm{C}$ are those at $\mathrm{PA}=173^{\circ}, 31^{\circ}$, and $49^{\circ}$, respectively. It is from these slit positions that the P-V arrays were simulated in Figs. 2-4. The overlaid boxes cover the knots used in the same figures.

is that they are candidates for type Ia supernova progenitors (Townsley \& Bildsten 2005) along with their recurrent counterparts (e.g. T. Crb as discussed in Hillman et al. 2016). Old nova shells give insight into dust production, clumping, and ISM dispersal mechanisms, thus they should be understood at all evolutionary phases.

There are many unknowns surrounding the origin of the morphology of nova shells such as whether their ejection is spherically uniform or intrinsically bipolar, (e.g. Porter et al. 1998; Lloyd et al. 1997). There is recent evidence in the case of V339 Del that shaping of ejected material happens very early on; non-sphericity is evident in the first days after outburst (Schaefer et al. 2014). The common envelope phase is thought to play a major role in the shaping of nova remnants, and planetary nebulae alike (Balick 1994; Nordhaus \& Blackman 2006). Slower nova events (regarding both photometric evolution and ejection velocities) are believed to have stronger deviations from spherical symmetry. This hints at the importance of the role the common envelope phase plays, when the time spent by the binary in the envelope is longer than their orbital period. Shaping is also expected from interaction with prior ejecta, which would also be non-spherical owing to various similar processes to the nova ejecta. There is observational evidence of dependence of axial ratio on speed class that can be seen in Fig. 8 of Slavin et al. (1995).

As an old, bright, and close nova shell with published kinematics, GK Per is an ideal object to study and enrich our knowledge regarding the mechanisms at work in a nova system, see Fig. 1. The 1901 GK Per nova event was possibly its first (Bode et al. 2004) and it has emerged as a well-studied and peculiar object. The central system has changed state, in line with hibernation theory (Shara 1981) although sooner than the theory predicts. It was the first classical nova remnant discovered in X-rays (Balman 2005) and non-thermal radio emission (Seaquist et al. 1989), implying an interaction with pre-existing material surrounding the system. Bode et al. (1987) first observed that a probable planetary nebula surrounds GK Per and thus is a likely candidate for the preexisting material detected in radio and X-rays. The spectacular superluminal light-echos by Wolf, Perrine and Ritchey in the years directly following the nova event (explained by Couderc 1939, as the forward scattering of light along dust sheets) reveal an abundance of material in the vicinity of the system. The first direct image of the nebulosity associated with the classical nova event was taken in 1916 (Barnard 1916).

GK Per has the longest period classical nova progenitor binary known to date, see Table 1, with a carbon deficient secondary star (Harrison \& Hamilton 2015). After undergoing several dwarf nova outbursts, observed since 1963, the object has been reclassified as an intermediate polar, which implies the presence of a strong magnetic field (1-10 megagauss, Watson et al. 1985). The dwarf nova outbursts on GK Per were first observed in 1963 and have a recurrence timescale of $3 \mathrm{yr}$ and a duration of 2 to 3 months. However, strong optical outbursts can be traced back to 1948 in the AAVSO data, once the central system had settled down to its quiescent state. Two more outbursts followed the 1948 explosion in quick succession, one in 1949 and another in 1950 (Sabbadin \& Bianchini 1983).

The central system is seen drifting through the local environment at about $45 \mathrm{~km} \mathrm{~s}^{-1}$, a value derived from proper motion studies (Bode et al. 2004). X-ray and radio observations reveal evidence of interaction between the SW quadrant of the shell with pre-existing material (Anupama \& Kantharia 2005; Balman 2005; Takei et al. 2015). The apparent box-like appearance of the nebula has long been observed (e.g. Seaquist et al. 1989). In the past arguments for flattening of the southern part of the shell through interaction with pre-existing material would not explain the flattened northern part of the shell. There have, however, been hints of an intrinsic symmetry to the nova shell such as the prolate structure proposed in Duerbeck \& Seitter (1987). They suggested a broken prolate structure, a missing southern cap, and an extended northern cap spanning the position angles (PA) 130-300 . Lawrence et al. (1995) conducted three-dimensional Fabry-Perot imaging spectroscopy of GK Per's nova shell, where they presented channel maps and a spatial model.

It had been previously believed that the expanding nova remnant was decelerating at a faster rate, based on rigorous analysis of proper motions and radial velocities of individual knots (Liimets et al. 2012, hereafter L12) came to the conclusion that the system is decelerating at a rate of at least 3.8 times slower than that derived by Duerbeck (1987). An eventual circularisation of the shell was proposed in L12. From considerations of the data presented in L12 as well as others, such as Duerbeck \& Seitter (1987), Lawrence et al. (1995), Tweedy (1995) and Shara et al. (2012), the opportunity to explore the structure and kinematics of the GK Per nova shell was undertaken. Knots associated with the shell are expanding almost radially away from the central system and have been followed over the decades; high-quality spectroscopy is also available. New data were collected to complement those found in the archives. GK Per exhibits a distinct arc of emission to the NE of the nova shell that is reminiscent of a jet but whose origin has not been settled (Bode et al. 2004; Shara et al. 2012). This study aims to determine the relationship between the expanding knotty debris, 
Table 1. GK Per, characteristics of the central binary system.

\begin{tabular}{lccccc}
\hline \hline Orb T $^{1}$ & WD T & $\mathrm{e}^{2}$ & $\operatorname{Inc}\left({ }^{\circ}\right)^{3}$ & $M_{1}{ }^{4}$ & $M_{2}{ }^{5}$ \\
\hline $1.997 \mathrm{~d}^{a}$ & $351 \mathrm{~s}^{b}$ & $0.4^{c} \| 1^{a}$ & $50-73^{d}$ & $0.9 M_{\odot}{ }^{a}$ & $0.25 M_{\odot}{ }^{a}$ \\
\hline
\end{tabular}

Notes. ${ }^{(1)} \mathrm{T}=$ Period; ${ }^{(2)} \mathrm{e}=$ ellipticity; ${ }^{(3)}$ inc = inclination; ${ }^{(4)} M_{1}=$ White dwarf mass; ${ }^{(5)} M_{2}=$ Mass of companion.

References. (a) (Crampton et al. 1986); (b) (Watson et al. 1985); ${ }^{(c)}$ (Kraft 1964); ${ }^{(d)}$ (Morales-Rueda et al. 2002).

the dwarf nova winds, the nature of the jet-like feature and the planetary nebula.

This paper follows the structure outlined here: observations, both archived and new, are presented in Sect. 2; in Sect. 3 the analysis of the observations is explained; a discussion follows in Sect. 4; and conclusions in Sect. 5.

\section{Observations}

\subsection{Imaging}

Using the $2.3 \mathrm{~m}$ Aristarchos telescope in Greece, new imaging was collected of GK Per on the 27 August 2014. The observations consisted of two narrowband filters focused on $\mathrm{H} \alpha$ and [N II] with exposures of $1800 \mathrm{~s}$ in each filter. The seeing was of the order of 2 arcsec. A $1024 \times 1024$ CCD detector was used where each $24 \mu \mathrm{m}$ square is $\equiv 0.28 \operatorname{arcsec}_{\text {pixel }}{ }^{-1}$ after $2 \times 2$ binning. The observations are summarised in Table $2 \mathrm{a}$. The imaging data were reduced and world coordinate system matching completed using standard routines in IRAF $^{1}$.

Narrowband filters, essential for deducing nebula structure have the disadvantage of missing emission of very explosive sources that is Doppler shifted out of the filter bandpass. The Aristarchos narrowband images ( $\mathrm{H} \alpha$ and $[\mathrm{N} \mathrm{II}])$, which were combined by scaling the flux of field stars to match and then used in the comparison Figs. 2-4, cover 6558.5A-6575.5 $\AA$ and 6579.5-6596.5 $\AA$, respectively, giving a contribution of over $4 \AA$ from $\mathrm{H} \alpha$. The information below $-200 \mathrm{~km} \mathrm{~s}^{-1}$ is therefore missing, but the information from +560 up to $+740 \mathrm{~km} \mathrm{~s}^{-1}$ is accounted for by the $6548 \AA$ emission, which is about four times weaker than its $6583 \AA$ counterpart, but stronger than $\mathrm{H} \alpha$. For $6583 \AA[\mathrm{N} \mathrm{II}]$ we miss data from -160 to $-340 \mathrm{~km} \mathrm{~s}^{-1}$ and +610 . From the observations, $\mathrm{H} \alpha$ and [N II] have the strongest emission in the nova shell and together we obtain contributions from all knots except those from -200 to $-340 \mathrm{~km} \mathrm{~s}^{-1}$. Overall, in the $-1000 \mathrm{~km} \mathrm{~s}^{-1}$ to $+1000 \mathrm{~km} \mathrm{~s}^{-1}$ range we are missing $7 \%$ of the information on the knots comprising the nova shell at these constraints. The coverage also shows higher velocity knots down to $-1100 \mathrm{~km} \mathrm{~s}^{-1}$ and up to $1500 \mathrm{~km} \mathrm{~s}^{-1}$. Owing to the variable emission between the lines covered in the Aristarchos observations and to differences in sensitivity, caution is to be exercised when judging differences in knots between these observations and those taken with the NOT + Mayall telescopes, see Figs. 2-4. The degraded seeing conditions of the Aristarchos telescope observations would be expected to mostly compensate for the missing information.

1 IRAF is distributed by the National Optical Astronomy Observatories, which are operated by the Association of Universities for Research in Astronomy, Inc., under cooperative agreement with the National Science Foundation.

\subsection{Spectroscopy}

Echelle spectroscopic data were also obtained in order to measure the extinction velocities of several interesting knots, to build a more complete view of the remnant, and to examine the jetlike feature. These were obtained using the Manchester Echelle Spectrometer (MES) instrument at the San Pedro Mártir (SPM) observatory in Mexico (Meaburn et al. 2003). The data were collected over the course of two observing periods, November 2014 and March 2015, and in three different positions. The slit positions, overlaid on an image of the system in Fig. 1, were observed with the instrumentation in its $f / 7.5$ configuration. A Marconi $2048 \times 2048$ CCD was used with a resultant spatial resolution $\equiv 0.35$ arcsec pixel ${ }^{-1}$ after $2 \times 2$ binning was applied during the observations with the $\sim 6^{\prime}$ slit. Bandwidth filters of 90 and $60 \AA$ were used to isolate the 87 th and 113th orders containing the $\mathrm{H} \alpha+[\mathrm{N}$ II] $\lambda \lambda 6548,6583$ and [O III] $\lambda 5007$ nebular emission lines.

\subsection{Archival data}

Archival imaging and spectroscopy from different epochs and telescopes were obtained from the NOAO, NOT, IRSA, and MAST databases. The archival observations are from the $\mathrm{Hub}$ ble Space Telescope, the WISE satellite, the Nordic Optical Telescope and the Mayall telescope, and are summarised in Table $2 b$. The principal data sets used were Mayall and HST observations reported by (Shara et al. 2012, hereafter S12), NOT data by L12, and WISE nova data first studied in Evans et al. (2014). Further details and analyses of these data are contained in these references. Here the data were collated and reduced in the same manner as the more recent MES and Aristarchos observations in IRAF.

Archival echelle spectroscopy bi-dimensional line profiles or position-velocity (P-V) arrays were generated for the six ALFOSC position angles listed in Table $2 \mathrm{~b}$. For each PA there were between four and eight individual knots or knot complexes, for which $\mathrm{P}-\mathrm{V}$ information could be subtracted. The P-V arrays were created by comparing rest wavelengths to the position of the strongest spectral lines of wavelength calibrated spectra. In this way velocity scales were made available for subsequent analysis using the Doppler-redshift relation.

\section{Analysis}

The P-V arrays were mostly created from archival ALFOSC data and for one position angle they are complemented by more recent echelle spectra from MES-SPM $\left(\mathrm{PA}=30^{\circ}\right.$ and $31^{\circ}$, respectively, see Table 2 and Fig. 1), allowing for constraints to be made on spatio-kinematics of individual systems. The P-V arrays were simulated using the morpho-kinematic code SHAPE (Steffen et al. 2011) ${ }^{2}$.

Modelling with SHAPE is carried out on different spatial scales. The nova shell as directly modelled from observations of the knots is described first in Sect. 3.1. Section 3.2 explores the structure of the overall distribution of the knots and Sect. 3.3 reveals new results on the jet-like feature associated with the remnant. 2 A full discussion on SHAPE modifiers can be found at http://
bufadora.astrosen. unam.mx/shape/ 
Table 2. Observations.

(a)

\begin{tabular}{|c|c|c|c|c|c|c|}
\hline Date & Type & Telescope & Instrument & Filter CWL/FW( $(\AA)$ & $\mathrm{PA}^{*}$ & Exp. (s) \\
\hline \multirow[t]{2}{*}{ 2014-Aug-27 } & Imaging & Aristarchos $^{1}-2.3 \mathrm{~m}$ & LN CCD & $\mathrm{H} \alpha 6567 / 17$ & & 1800 \\
\hline & Imaging & Aristarchos $-2.3 \mathrm{~m}$ & LN CCD & [N II] 6588/17 & & 1800 \\
\hline \multirow[t]{4}{*}{ 2014-Nov-28 } & Spectroscopy & $\mathrm{SPM}^{2}-2.12 \mathrm{~m}$ & MES & $\mathrm{H} \alpha+[\mathrm{N} \mathrm{II]} 6541 / 90$ & $45^{\circ}$ & 1800 \\
\hline & Spectroscopy & $\mathrm{SPM}-2.12 \mathrm{~m}$ & MES & $\mathrm{H} \alpha+[\mathrm{N} \mathrm{II]} 6541 / 90$ & $30^{\circ}$ & 1800 \\
\hline & Spectroscopy & $\mathrm{SPM}-2.12 \mathrm{~m}$ & MES & [O III] 4984/60 & $45^{\circ}$ & 1800 \\
\hline & Spectroscopy & $\mathrm{SPM}-2.12 \mathrm{~m}$ & MES & [O III] 4984/60 & $30^{\circ}$ & 1800 \\
\hline 2015-Mar-27 & Spectroscopy & SPM $-2.12 \mathrm{~m}$ & MES & $\mathrm{H} \alpha+[\mathrm{N} \mathrm{II}] 6541 / 90$ & $9^{\circ}$ & 1800 \\
\hline \multirow[t]{2}{*}{ 2015-Mar-28 } & Spectroscopy & $\mathrm{SPM}-2.12 \mathrm{~m}$ & MES & $\mathrm{H} \alpha+[\mathrm{N} \mathrm{II}] 6541 / 90$ & $9^{\circ}$ & 1800 \\
\hline & Spectroscopy & $\mathrm{SPM}-2.12 \mathrm{~m}$ & MES & [O III] 4984/60 & $9^{\circ}$ & 1800 \\
\hline \multicolumn{7}{|l|}{ (b) } \\
\hline Date & Type & Telescope & Instrument & Filter/grism CWL/FW(Å) & PA & Exp. (s) \\
\hline 1995-Nov-8 & Imaging & $\mathrm{HST}^{3}-2.4 \mathrm{~m}$ & WFPC2 & F658N 6591/29 & & 1400 \\
\hline 1997-Aug-1 & Imaging & $\mathrm{HST}^{3}-2.4 \mathrm{~m}$ & WFPC2 & F658N 6591/29 & & 1200 \\
\hline 2007-Sep-3/5 & Imaging & $\mathrm{NOT}^{4}-2.5 \mathrm{~m}$ & ALFOSC & $\mathrm{H} \alpha 6577 / 180$ & & 3600 \\
\hline 2010-Feb-6/7 & Imaging & $\mathrm{KPNO}^{5}-4 \mathrm{~m}$ & CCD Mosaic & $\mathrm{H} \alpha 6563 / 80$ & & 7440 \\
\hline 2010-Feb-12 & Imaging & $\mathrm{WISE}^{6}-40 \mathrm{~cm}$ & Survey Camera & $\mathrm{B} 3(12 \mu \mathrm{m})^{7}$ & & 4422 \\
\hline 2007-Sep-3 & Spectroscopy & NOT $-2.5 \mathrm{~m}$ & ALFOSC & $\begin{array}{l}\text { g17 6600/500 } \\
\end{array}$ & $31^{\circ}$ & 3600 \\
\hline \multirow[t]{3}{*}{ 2007-Sep-4 } & Spectroscopy & NOT $-2.5 \mathrm{~m}$ & ALFOSC & g17 6600/500 & $86^{\circ}$ & 1800 \\
\hline & Spectroscopy & $\mathrm{NOT}-2.5 \mathrm{~m}$ & ALFOSC & g17 6600/500 & $107^{\circ}$ & 1500 \\
\hline & Spectroscopy & $\mathrm{NOT}-2.5 \mathrm{~m}$ & ALFOSC & g17 6600/500 & $312^{\circ}$ & 1800 \\
\hline \multirow[t]{2}{*}{ 2007-Sep-5 } & Spectroscopy & $\mathrm{NOT}-2.5 \mathrm{~m}$ & ALFOSC & g17 6600/500 & $49^{\circ}$ & 1800 \\
\hline & Spectroscopy & $\mathrm{NOT}-2.5 \mathrm{~m}$ & ALFOSC & g17 6600/500 & $173^{\circ}$ & 1800 \\
\hline 2010-Feb-10 & Spectroscopy & KPNO - $4 \mathrm{~m}$ & RC-Spec & BL400 5500/7000 & $89.7^{\circ}$ & 9600 \\
\hline
\end{tabular}

Notes. (a) Are newly acquired for this work whereas (b) were obtained from data archives. ${ }^{(1)}$ Aristarchos Telescope, Helmos Observatory; (2) San Pedro Mártir Observatory; ${ }^{(3)}$ Hubble Space Telescope; ${ }^{(4)}$ Nordic Optical Telescope; ${ }^{(5)}$ Mayall Telescope, Kitt Peak National Observatory;

(6) Wide-field Infrared Survey Explorer; ${ }^{(7)}$ WISE band 3 has a resolution of 6.5". ${ }^{(*)} \mathrm{PA}=$ Position Angle.

\subsection{Modelling individual knots}

In the first instance, a direct model from published data associated with L12 was made. For this initial model, 115 knots (from the online table of L12) with the most substantial data were modelled as individual cylinders with expansion velocities, distance from the central star, PA, and inclinations derived using the prescriptions of L12. In order to achieve this, each individual knot was modelled as a cylinder with $x, y, z$ positions in arcseconds specified with the translation modifier; the PA and inclination were replicated using a rotation modifier; and the expansion velocities specified explicitly with the velocity modifier. We note the modelled microstructure in panel (c) Fig. 6. Later a radial velocity component was added to knots to match the ALFOSC $\mathrm{P}-\mathrm{V}$ arrays in Figs. 2-4. Irregularities in knot structure were then simulated by modifying the cylinder primitives to match their respective P-V arrays using bump, stretch, and squish modifiers. For another recent application and further explanation of these SHAPE modifiers see Clyne et al. (2015).

High-resolution images from several time frames were analysed in order to gain further knowledge on the interaction and fragmentation of clumps. Variations in the knot morphology and velocity distribution between different clump systems can be seen in Figs. 2-5. The long-slit spectroscopy discussed in L12 was re-analysed with a different intent, with the addition of our new data. As L12 derived the kinematics based on single
Gaussian peaks, we aimed to delve further and to examine the extended profiles of individual knots. To gain an understanding of the knot kinematics and morphology, spatially oriented P-V arrays were created using IRAF and OCTAVE (Eaton et al. 2014). The P-V arrays were difficult to reproduce with SHAPE owing to the amorphous nature intrinsic to the knots and the complex velocity field in which they reside.

Possible mechanisms were explored to explain the irregularities observed in the individual knot shapes, including dynamical instabilities with a main contributor thought to be the fast periodic winds produced by the frequent dwarf nova episodes. Interestingly, clumping of the ejecta began before the dwarf nova episodes had been observed. The initial clumping was most likely due to early interacting winds (Balman 2005). In Fig. 2 extended velocity-tails of individual knots are apparent; these tails appear wavy, which indicates their complex velocity structure. The longest tails are closer to the central system in the plane of the sky (the lower row in Fig. 2 is closer to the central star in the plane of the sky than the upper row and thus has a longer tail in velocity space since more velocity information is contained in these knots owing to their inclination towards the observer), hinting that their true structure is similar to those with "wavy" tails attributed in this work to shaping by dwarf nova winds (see Sect. 4). The longer tails present towards the central system was shown previously by L12, see their Fig. 8, where they 
mention that this could be a projection effect. Looking at Fig. 3, the first row demonstrates overlapping of knots that can be separated by morpho-kinematic modelling, whereas the second row demonstrates clear evidence of a bow-shock and low expansionvelocities also attributable to interaction between knots.

The difference in the P-V arrays in Fig. 4 over the seven year interval appear significant. The MES data has higher velocity resolution $\left(22 \mathrm{~km} \mathrm{~s}^{-1}\right.$ versus $32 \mathrm{~km} \mathrm{~s}^{-1}$ ), but lower sensitivity, see Table 2. As can be seen in Fig. 4, top row of panels, the ALFOSC P-V array and MES P-V array show differences in position as the proper motion and velocity-structure differences are due to a combination of different sensitivity and resolution, and are also due to a slight $\left(1^{\circ}\right)$ difference in the slit positions. However, the $1^{\circ}$ difference in position angle gives a separation of the slits of around $1^{\prime \prime}$ at a distance of $60^{\prime \prime}$ from the central system, which is within the seeing of both sets of observations. In addition the MES slit is 3.8" wide on the plane of the sky. The pattern of a bow shock around one or more knots remains evident with comparison to the SHAPE model, see the bottom row of Fig. 3 and the top row of Fig. 4. Also, in Fig. 3, the knots marked "X" are seen to undergo shaping changes and bifurcation over the time interval of the presented observations. Tracing these knots back to the HST imaging campaign of S12 they are seen to separate from the knot complex covered by the slit in the same panels as knot " $X$ ". In Fig. 2 the knot at the upper panel appears to have a tail that is bending back; this knot has a radial velocity of about $-650 \mathrm{~km} \mathrm{~s}^{-1}$ and the knot possibly associated with it to the left has a radial velocity of $-570 \mathrm{~km} \mathrm{~s}^{-1} 31^{\circ}$ in the ALFOSC long-slit observation and $-530 \mathrm{~km} \mathrm{~s}^{-1}$ seven years later in the MES data, see knot "c" in Fig. 5 and Table 4, which may be indicative of knot changes due to the complex dynamics of the environment. We note that Fig. 5 is complemented by Table 4 where the velocity widths and signal-to-noise ratios of the observations are shown.

We also note that the analysis of observation leads to a small number of knots ( 8 out of 115) that have large uncertainties in their deprojected true positions within the population of knots; these uncertainties are caused by large relative errors in one or more components of the deprojected distance estimates, or else are ballistic knots like those discussed in S12.

Examination of the radial velocity distribution with respect to $x, y$ positions of the knots is suggestive of an overall symmetry in the total distribution. The low-resolution channel maps of Lawrence et al. (1995) show similar symmetry on reinspection, see Fig. 7.

\subsection{Overall distribution}

The second modelling approach involved testing of basic shapes to fit the overall distribution of the knots with $x-y$ positions and radial velocity measurements; the sample size was increased to 148 through the inclusion of knots covered in our new observations, see the table in the Appendix. As an example of the versatility of this approach consider a sphere with a thin shell and the knots added by means of a texture modifier given the appropriate filling factor. The positions of the knots can be matched by adjusting their position on the shell so that the model and actual P-V arrays agree. The sphere can be deformed appropriately to simulate the somewhat box-like appearance of the remnant, or it can also be manipulated to have a bipolar, prolate or oblate shape. Also, the size of the structure can be easily modified to see if the same shape can match that of previous imaging campaigns, such as that of Seaquist et al. (1989). Comparison of the brightness distribution between epochs give clues on
Table 3. Best fit SHAPE orientation and velocity model parameters of the GK Per nova shell.

\begin{tabular}{lccccccc}
\hline \hline & $\begin{array}{c}\text { PA } \\
\left({ }^{\circ}\right)\end{array}$ & $\begin{array}{c}\text { Inc } \\
\left({ }^{\circ}\right)\end{array}$ & $\begin{array}{c}R_{\text {in }} \\
(\mathrm{pc})\end{array}$ & $\begin{array}{c}R_{\text {out }} \\
(\mathrm{pc})\end{array}$ & $\begin{array}{c}L \\
(\mathrm{pc})\end{array}$ & $\begin{array}{c}D \\
(\mathrm{pc})\end{array}$ & $\begin{array}{c}V_{\exp } \\
\left(\mathrm{km} \mathrm{s}^{-1}\right)\end{array}$ \\
\hline $\mathrm{O}$ & 120 & 54 & 0.093 & 0.109 & 0.125 & 0.093 & 1000 \\
$\mathrm{E}$ & 120 & 54 & 0.027 & 0.091 & 0.027 & 0.080 & 750 \\
$\mathrm{~W}$ & 120 & 126 & 0.027 & 0.091 & 0.027 & 0.080 & 750 \\
\hline
\end{tabular}

Notes. The size of the shell features are normalised to the shell's size during the 2007 observations of L12. PA = Position Angle, inc = inclination, $R_{\mathrm{in}}=$ inner radius of feature, $R_{\text {out }}=$ outer radius of feature, $\mathrm{L}=$ length, $\mathrm{D}=$ distance to central binary, $V_{\exp }=$ expansion velocity. With regards to the features $\mathrm{O}=$ outer cylinder, $\mathrm{E}=$ eastern cone, $\mathrm{W}=$ western cone.

Table 4. Comparison of RV profiles of the five brightest knots in common of the 30 and 31 slits. ALF = ALFOSC on the NOT telescope, MES at San Pedro Martir.

\begin{tabular}{lllllll}
\hline \hline Knot & $\begin{array}{l}F W H M \\
\mathrm{~km} \mathrm{~s}^{-1} \\
\text { MES }\end{array}$ & $\begin{array}{l}F W H M \\
\mathrm{~km} \mathrm{~s}^{-1} \\
\text { ALF }\end{array}$ & $\begin{array}{l}F W 10 \% \\
\text { MES }\end{array}$ & $\begin{array}{l}F W 10 \% \\
\text { ALF }\end{array}$ & $\begin{array}{l}\text { SNR } \\
\text { MES }\end{array}$ & $\begin{array}{l}S N R \\
\text { ALF }\end{array}$ \\
\hline $\mathrm{a}$ & 112 & 114 & 297 & 214 & 102 & 106 \\
$\mathrm{~b}$ & 54 & 59 & 97 & 112 & 47 & 27 \\
$\mathrm{c}$ & 75 & 120 & 177 & 243 & 35 & 46 \\
$\mathrm{~d}$ & 116 & 92 & 266 & 154 & 26 & 42 \\
$\mathrm{e}$ & 164 & 106 & 231 & 305 & 81 & 94 \\
\hline
\end{tabular}

the sequence of which components and sides started becoming shock illuminated. However, a sphere primitive was found to be unable to reproduce the channel maps of Lawrence et al. (1995).

This second approach was then applied to several common nova shell morphologies that can be manipulated in the same ways as the sphere. To achieve the best fit an inclination, PA, and expansion velocity were specified (see Table 3 ) implemented using the rotation and velocity modifiers in SHAPE. To simulate the knots a texture modifier was added and the knots were projected radially through the thickness of the cylindrical shell. Structure was added subsequently to the cylindrical model to account for spatial and Doppler shift irregularities. This structure consists of polar cones, see Fig. 6 and Table 3, the polar features have low equatorial velocities and higher polar velocities.

Previous efforts towards the understanding of the shell structure were unable to derive the nova shell's true morphology; the shell is almost consistently referred to as roughly circular and asymmetric. The flattened SW part of the shell has been given much attention since it is the location of interaction with pre-existing material; however, previous authors failed to address the flattened NE part of the shell, although it is remarked upon in several works, e.g. Lawrence et al. (1995). L12 argues for the eventual circularisation of the shell; however, here we find that the main shell most probably consists of a barrel-like equatorial structure (Fig. 6, Table 3) and will therefore not experience such a progression towards spherical symmetry. The position angle derived for the nova shell here fits that of the fossil bipolar planetary nebula (Scott et al. 1994). In the literature Duerbeck \& Seitter (1987) provided a symmetric model for the morphology by using a similar methodology to that employed here. Their model has a prolate structure, but we find an axial ratio closer to $1: 1$. More importantly we find that the equatorial and polar regions are perpendicular to the orientation suggested by Duerbeck \& Seitter (1987), Seaquist et al. (1989), and 

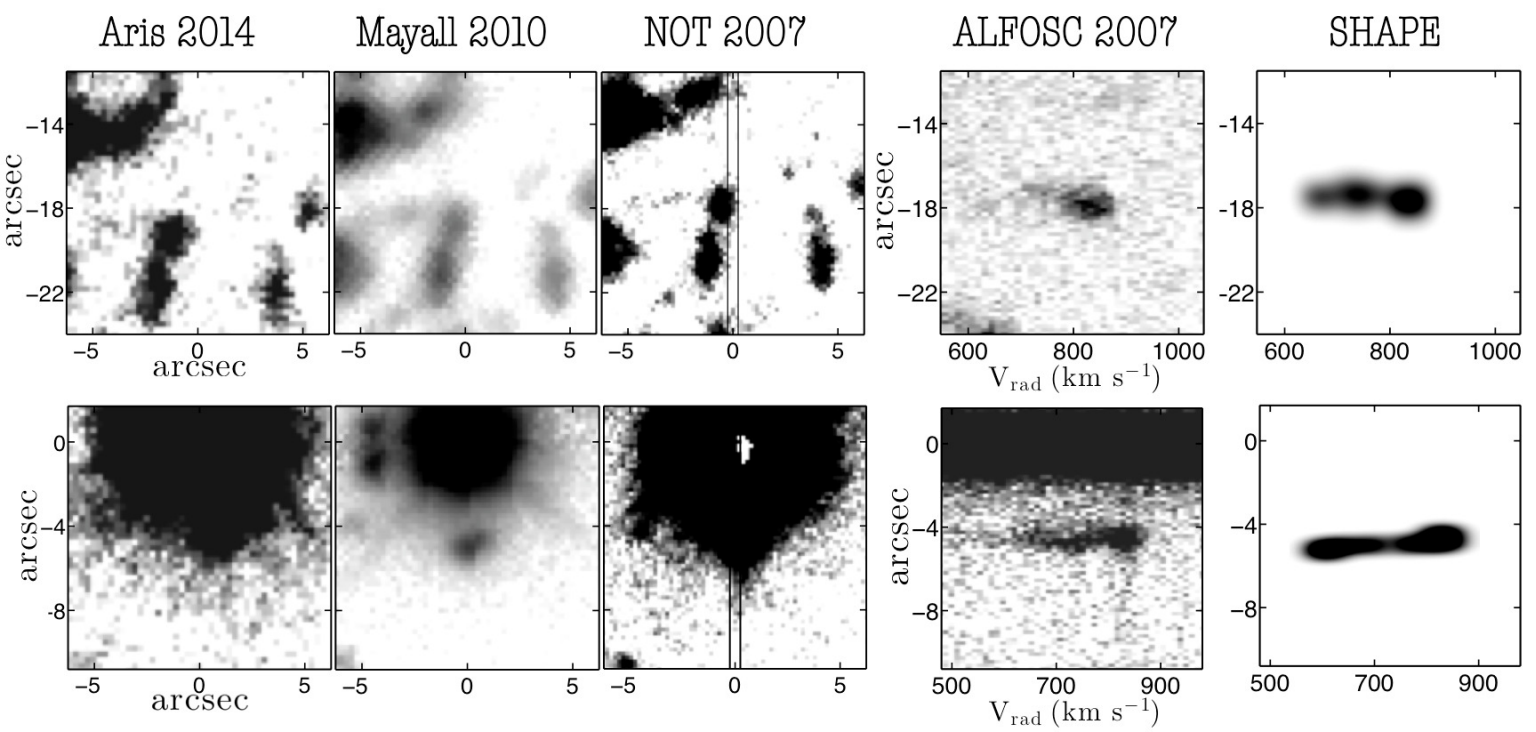

Fig. 2. Examples of single knots progressing over time (2007-2014) and their corresponding P-V array and morpho-kinematic SHAPE. Spatial extent is 12.5 arcsec on each side, and the velocity ranges are $500 \mathrm{~km} \mathrm{~s}^{-1}$. On the NOT panels the overlaid slit width and position is shown. The Aristarchos and NOT panels in the bottom row are contaminated by the central star leading to a very strong contrast between knots and star. Here, and in Figs. 3 and 4 the displayed knots are positioned along the observed slit axis highlighted in Fig. 1, where the top row corresponds to a slit position angle of $49^{\circ}$ and the bottom row to $173^{\circ}$. The spatial axis distance from the central star is positive towards the top of the slit and negative towards the bottom.
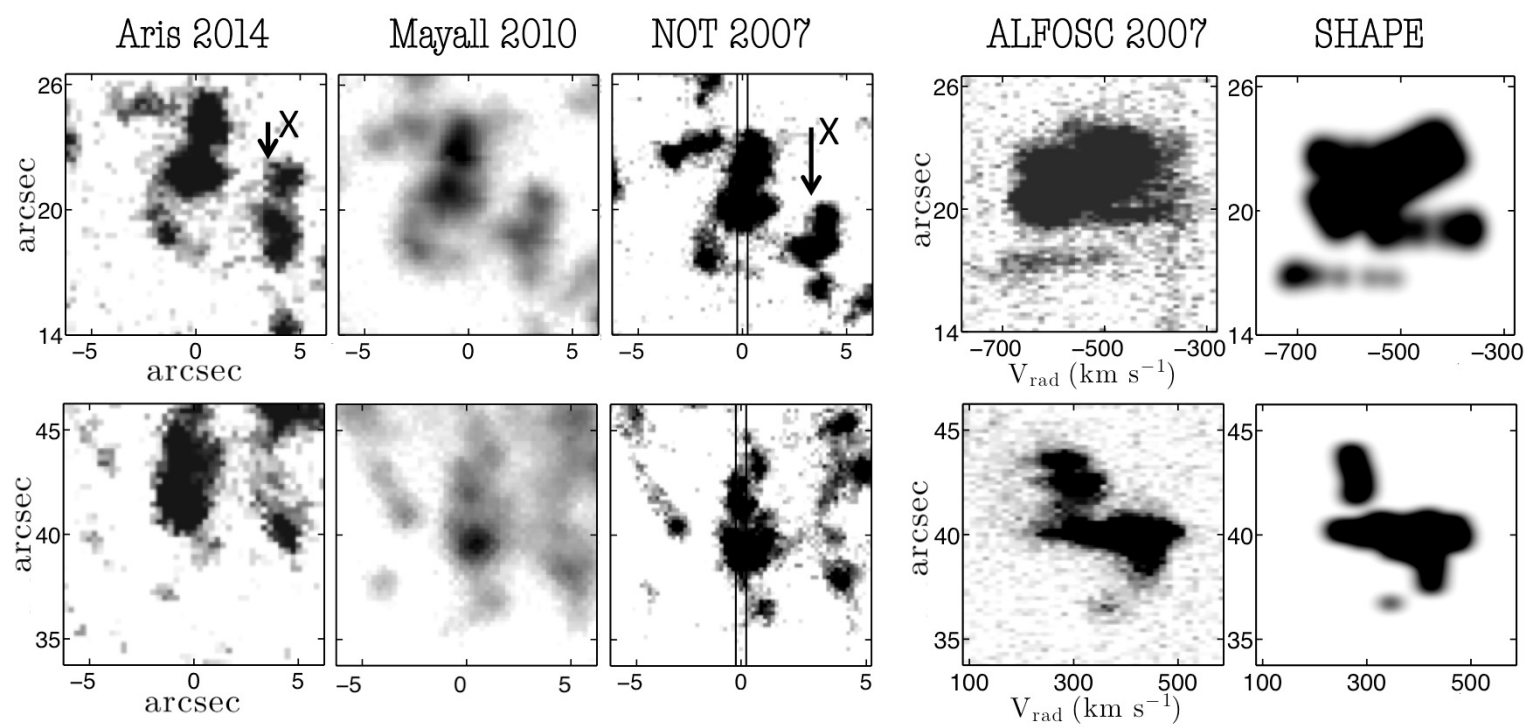

Fig. 3. Same as in Fig. 2 except with examples of more than one knot in each image. Spatial extent is 12.5 arcsec and velocity scales are $500 \mathrm{~km} \mathrm{~s}^{-1}$. The top row shows the separation of knots just to the bottom right of the multiple knots covered with the $173^{\circ}$ slit, the bottom row corresponds to a position angle $49^{\circ}$. The knot marked "X" can be seen to break away from the knot covered by the slit next to it in HST images from 1995 and 1997; the corresponding P-V array of this knot shows ongoing separation of knots in this clump.

Anupama \& Prabhu (1993). The newer data, however, firmly indicates that this previous model cannot fit the Doppler overall distribution of the knots or channel maps of Lawrence et al. (1995), see Fig. 7 for the fit of our model to their channel maps and Fig. 8 for an overlay of our model on their $z$ projection of the shell. Lawrence et al. (1995) interpreted the structure of GK Per as "approximately spherical (with) striking deviations in the symmetry". The aforementioned deviations were (i) a northern blue-shifted region; (ii) a low-radial-velocity "central" region and (iii) a small bulge to the south-southwest. These apparent irregularities can be explained within the context of our model as (i) the blue-shifted face of the cylinder; (ii) the northern and southern sides of the main barrel shell; and (iii) a combination of the eastern-polar-feature (blue-shifted knots) combined with some knots attributable to the main shell (those that are redshifted). A comparison of the fit of two separate proper motion studies of the knots associated with the shell (L12 and S12) can be seen in Fig. 9, and a comparison of the radial velocity measurements of L12 to our model can be seen in Fig. 10.

Referring to Fig. 6, several knots possibly associated with the western polar region of the oblate structure (i.e. along the minor, NW-SE, axis of the cylinder) have higher expansion velocities than our model suggests, which is a likely analogue to the diametrically opposed feature (iii) mentioned above. These features could be due to recent significant interaction of these knots with preexisting circumstellar material which is enhanced 

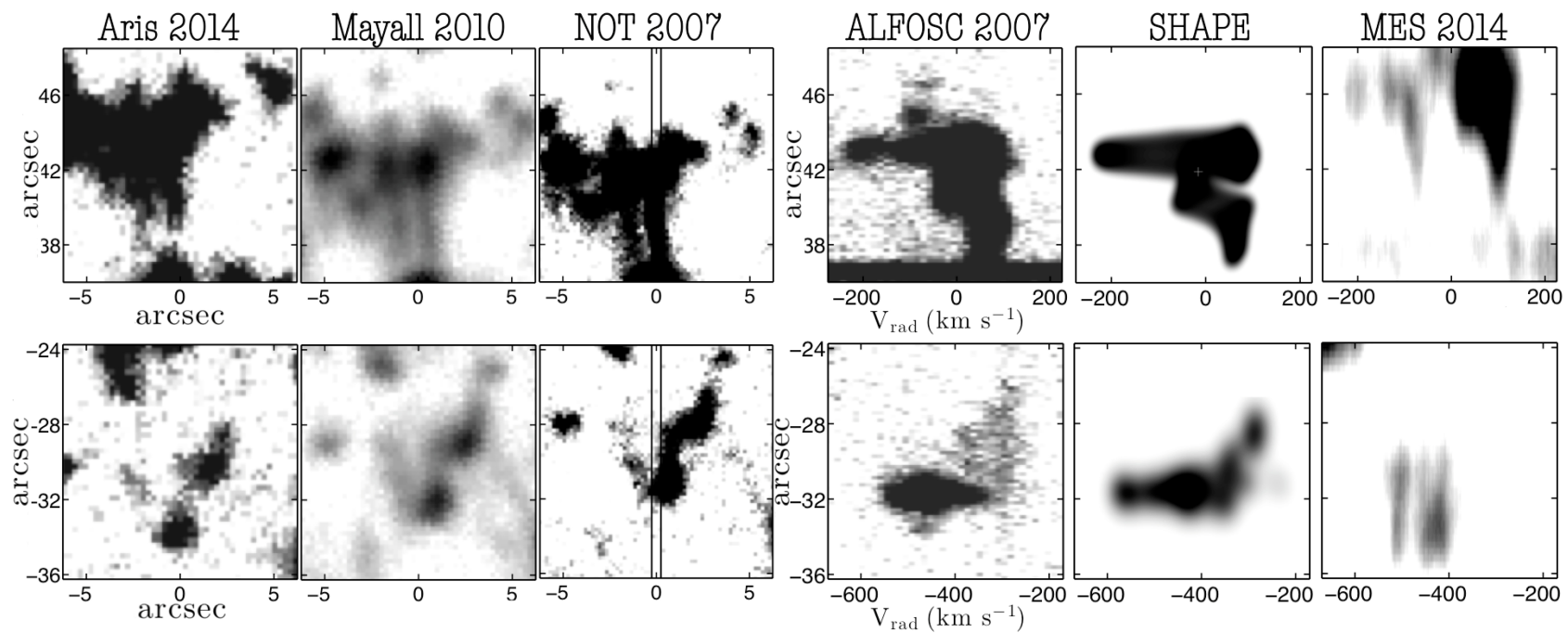

Fig. 4. Same as in Figs. 2 and 3 except with additional epoch P-V information. The knots here are along an axis of symmetry of the nova shell (slit position angles of $30^{\circ}$ and $31^{\circ}$ for the ALFOSC and MES observations respectively). The ALFOSC P-Vs correspond to the 2007 NOT image and the MES, [N II] $6583.39 \AA$, P-Vs correspond to the Aristarchos observations epoch.
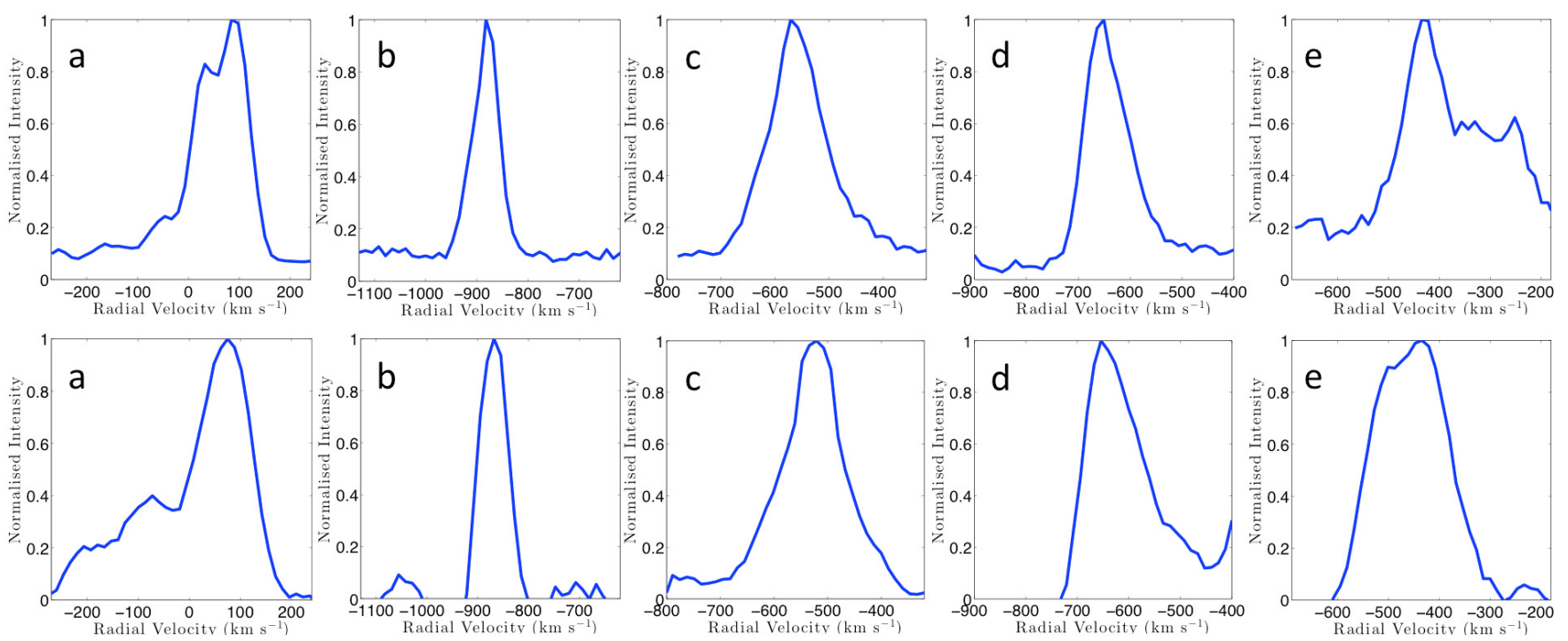

Fig. 5. Radial profiles of five bright knots from the ALFOSC slit with $\mathrm{PA}=31^{\circ}$ in the top row, and the corresponding MES knots with slit at $\mathrm{PA}=30^{\circ}$, in the bottom row. The knots displayed are seen in their [N II] 6583.39 A emission line, knot "a" corresponds to the top row of Fig. 4 and knot "e" to the bottom knot of the same figure.

in this direction (see Fig. 13 bottom panel). Finally, the inhomogeneous nature of the circumstellar matter evident in Fig. 13 gives a natural explanation as to why some knots in this direction are not significantly slowed and appear as outflows with large spatial displacements in Fig. 6c. Their displacement may also be apparent as the majority of such knots have substantial errors in their distance determinations.

\subsubsection{Criss-cross mapping}

Criss-cross mapping is a relatively new technique presented in Steffen \& Koning (2011) for the first time, see also Akras \& Steffen (2012). The technique leads to clues in the analysis of internal proper motions of gaseous nebulae by seeing where the proper motion vectors converge when stretched to infinity.
Applying this technique to the proper motion measurements in the online data table of L12, see Fig. 11, an apparent offset of about $2^{\prime \prime}$ to the north of the geometric centre of the nova shell is observed. Apart from the nice radial outflow seen in these maps, pairing Fig. 11 with the proper motion measurement of the central star of $0.015^{\prime \prime} \mathrm{yr}^{-1}$ (Bode et al. 2004), a kinematical age of $130 \mathrm{yr}$ is obtained for the offset of the geometrical centre in good agreement with the age of the shell. However, these results lie within the observational noise and should be taken with caution. Another interpretation for the geometrical offset is that the nova shell is partially bipolar and that the expansion is not exactly radial at mid-latitudes.

\subsection{Kinematics and imaging of the jet-like feature}

Deep imaging reveals a faint jet-like feature (Anupama \& Prabhu 1993) which protrudes from the NE of the nebula and stretches 

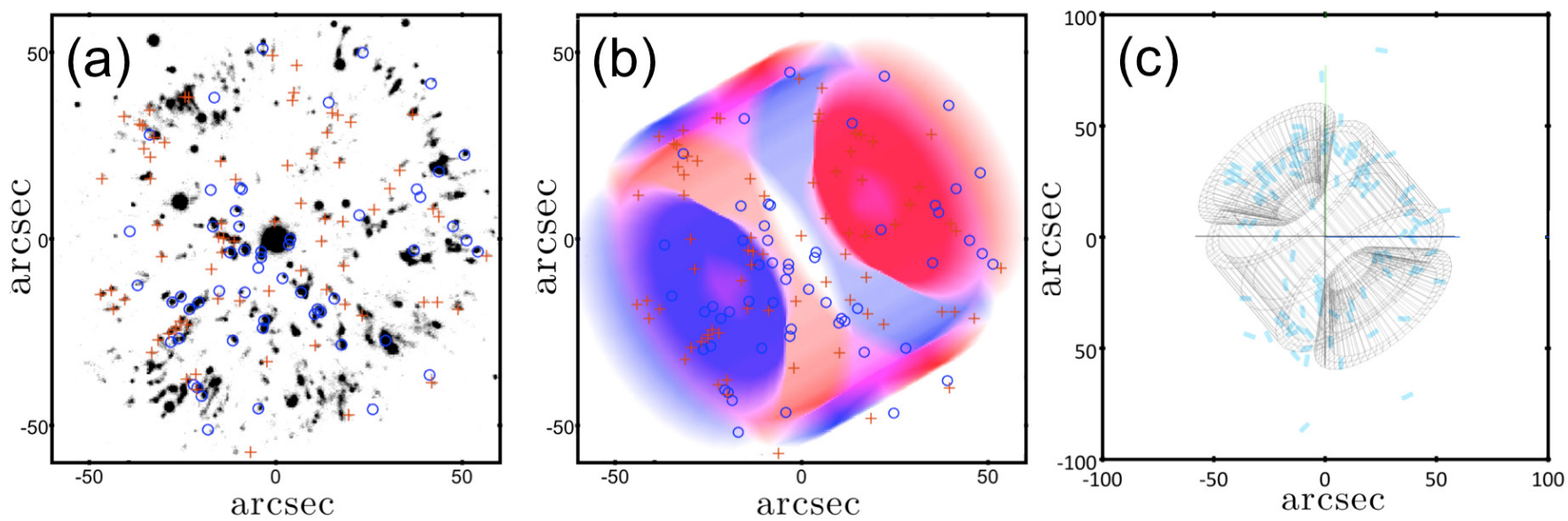

Fig. 6. Panel a): red-blue Doppler distribution from observed radial velocities of 148 knots overlaid on an image from the Mayall telescope; north is up and east is to the left. Panel b): fit of the cylinder on the observed radial velocity distribution. Panel c): model fit to the deprojected observations of L12 in the $x-z$ plane. We note the extended ballistic knots and the larger plotted area here.
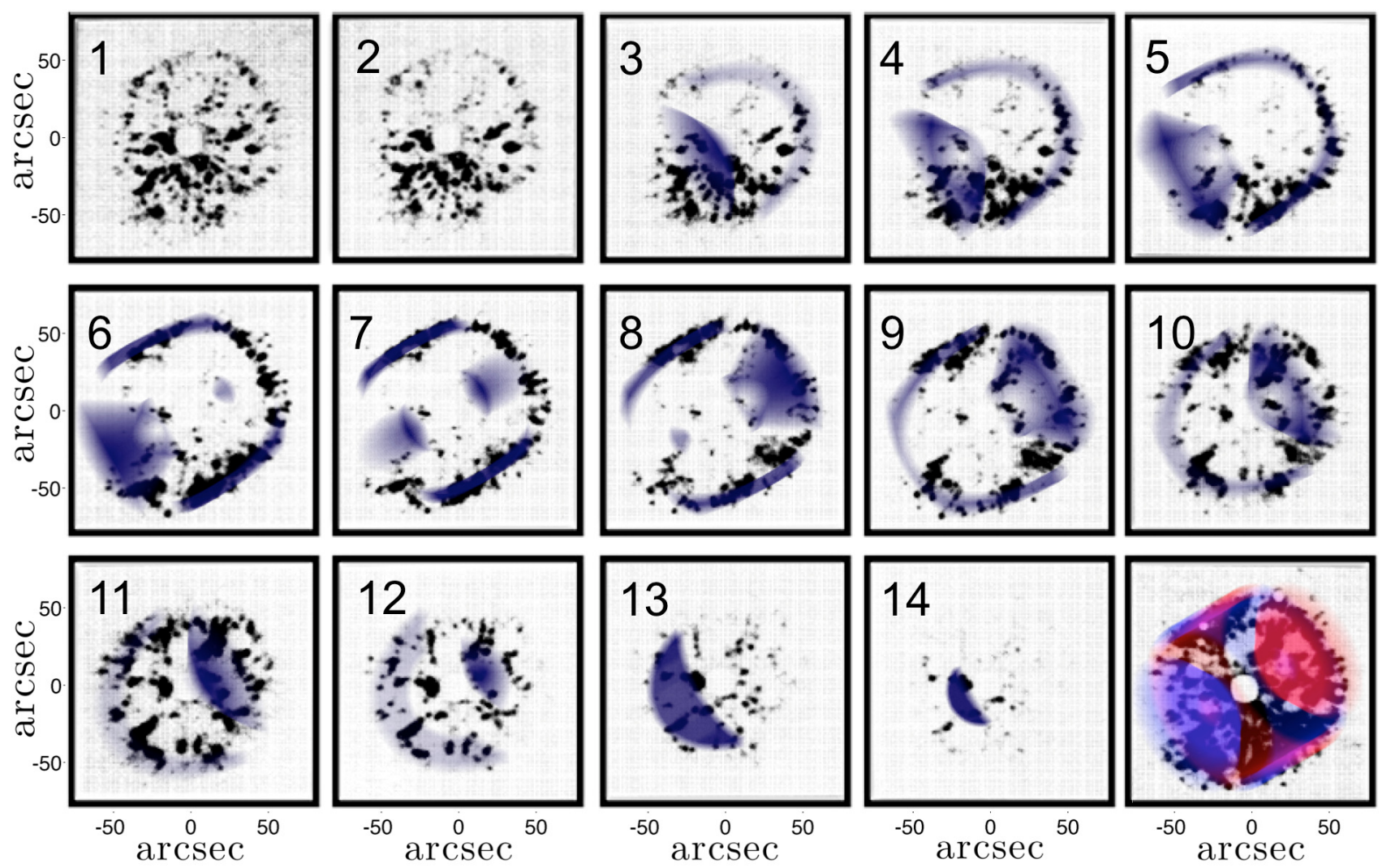

Fig. 7. Channel maps from Lawrence et al. (1995) (dots) compared to our scaled morpho-kinematic model (overlaid shadow). Frames 1-14 start at $-840 \mathrm{~km} \mathrm{~s}^{-1}$ and go up to $980 \mathrm{~km} \mathrm{~s}^{-1}$, the velocities being relative to the [N II] $6583 \AA$ emission line in steps of $140 \mathrm{~km} \mathrm{~s}^{-1}$ with a resolution of $240 \mathrm{~km} \mathrm{~s}^{-1}$. The first four frames have the red side dominated by $\mathrm{H} \alpha$ emission (the first two extremely so and whose SHAPE models have been left out for this reason) and the final frame is a sum of all those previously overlaid with the red-blue model distribution. Influence of the $\mathrm{H} \alpha$ emission is visible in the form of the persistent $\mathrm{S} / \mathrm{SE}$ feature up to frame 7.

out into finger-like structures until it eventually disappears, see Fig. 13, top panel. Previously there have been several theories for the origin of this enigmatic feature, (Anupama \& Kantharia 2005; Bode et al. 2004; Shara et al. 2012). Light-echo contours over IRAS imagery (Bode et al. 2004) hinted that it predated the nova shell. Shara et al. (2012) discussed three possible origins for this feature including a Mira-like tail (which they finally rule out), a collimated jet, and a rotating feature.
New observations presented here, corrected for the barycentric and heliocentric velocities towards the source, give averaged radial velocities over three intersecting slit position angles of 14 , 14,16 , and $17 \pm 22 \mathrm{~km} \mathrm{~s}^{-1}$ for the [O III] $5007 \AA$, [N II] $6548 \AA$, H $\alpha 6563 \AA$, and [N II] $6583 \AA$ emission lines, respectively. The full width at half maximum for each emission line is around $30 \mathrm{~km} \mathrm{~s}^{-1}$, see Fig. 12. Since our new kinematic data strongly suggest a low velocity for the jet, we put forward the idea 


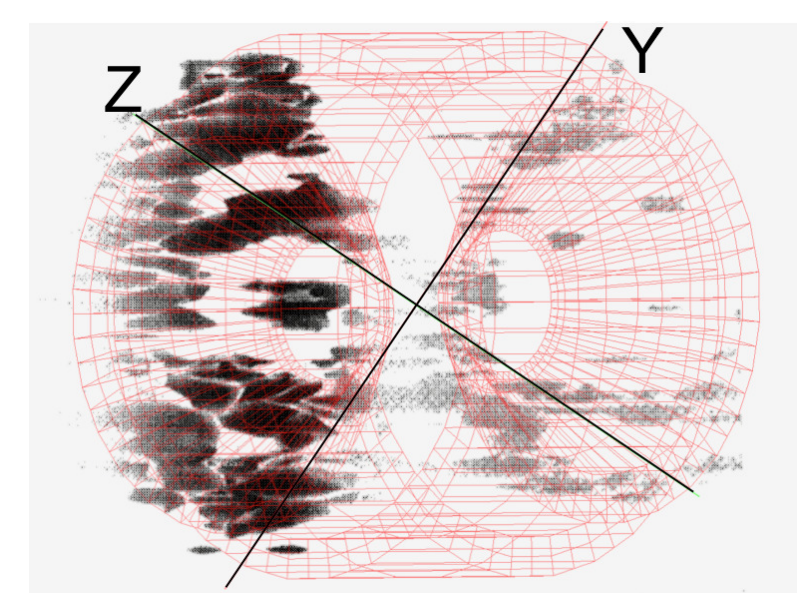

Fig. 8. $Z$ cut model presented by Lawrence et al. (1995; greyscale, adapted from their Fig. 6, panel C) with our model overlaid on the observations (the mesh). Here we see the model polar features trace out the morphology well.

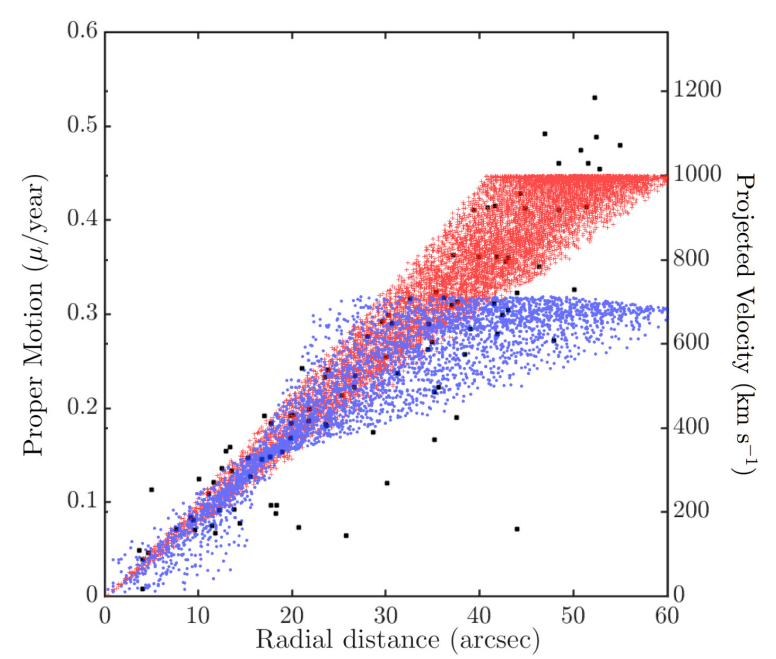

Fig. 9. Fit of the model presented here to the proper motion versus radial distance. The filled black squares are proper motion measurements from the online data table of L12 in terms of radial distance. The deviation from pure radial expansion is accounted for by the polar cones (blue overlay). The red corresponds to the cylinder fit to data presented in L12.

that the feature may be an illuminated section of the ancient planetary nebula. A well-explained nebula could naturally account for the curvature of the jet-like feature. Low-velocity features are regularly seen at the waist regions of extreme bipolar planetary nebulae, e.g. NGC 2346 (Kastner \& Gatley 2000). Evidence for the association of this feature with the planetary nebula shell is seen in the faint enhancement of the $\mathrm{H} \alpha$ and [O III] $5007 \AA$ lines at the interaction region to the SW of the nova shell, as noted by Anupama \& Kantharia (2005), Balman (2005), and Takei et al. (2015). In further support of this hypothesis Anupama \& Kantharia (2005) find emission extending from $20 \mathrm{~km} \mathrm{~s}^{-1}$ to $-25 \mathrm{~km} \mathrm{~s}^{-1}$ from the $21 \mathrm{~cm} \mathrm{HI}$ line, with emission near $-5 \mathrm{~km} \mathrm{~s}^{-1}$ west of the nova shell corresponding to the shell's "blue" side. Hinting that the "jet" belongs to the red-shifted eastern bipolar lobe of the ancient surrounding planetary nebula.

\section{Discussion}

The newly derived morphology of the nova shell in this work is consistent with observations of the GK Per system. One of

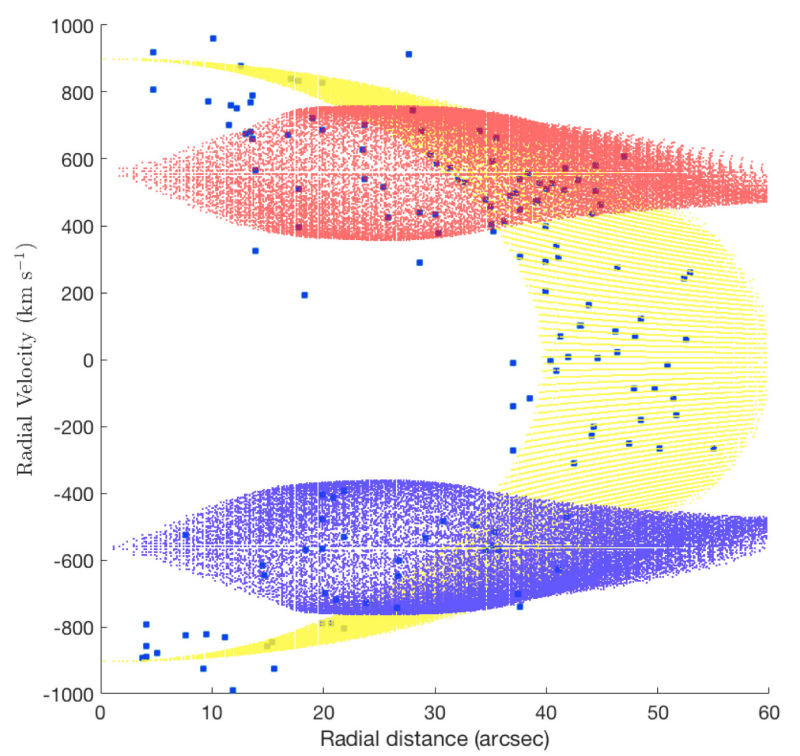

Fig. 10. Equivalent fit to Fig. 9, upper panel, in L12. The yellow represents the main cylindrical shell, whereas the red and the blue cover the polar features.

the main findings in L12 was that there was no significant deceleration of the knots in the SW quadrant of the nova shell, yet it had been long believed that the shell is experiencing a stronger interaction with circumbinary material in this quadrant (Duerbeck \& Seitter 1987), although L12 found a higher kinematical age for the NE part of the nova shell. The inclination $\left(54^{\circ}\right)$ and $\mathrm{PA}=120^{\circ}$ proposed here for the nova shell pose a problem if they are related to the position angle of the jet $\left(\mathrm{PA} \sim 30^{\circ}\right)$ or related to the orientation of the underlying binary. Even if we dismiss the PA for the shell in this work and consider the work of Bianchini et al. (1982), where they derived an inclination angle of $66^{\circ}$ for the accretion disk, then if the jet was indeed launched by the accretion disk larger radial velocity measurements would be expected rather than those of a typical planetary nebula. Concluding that the jet-like feature must simply be an illuminated part of the ancient planetary nebula. A contrast in the shape of the planetary nebula and nova shell would lend valuable clues to the efficiency of the underlying shaping mechanism.

As there has been some discussion on the effect of the surrounding pre-existing material on the shell morphology, the bullet crushing time (Redman et al. 2002; Poludnenko et al. 2004) was calculated based on density ratios and clump properties, see Fig. 9. The bullet crushing time is the hydrodynamical timescale of an individual knot $t_{\mathrm{d}}$ and is given by $\chi$, the density ratio of the circumbinary material to that of an individual clump, the clump radius $R_{\mathrm{c}}$, and the velocity of the clump moving through the local medium is $V_{\mathrm{s}}$ :

$t_{\mathrm{d}}=\chi \frac{R_{\mathrm{c}}}{V_{\mathrm{s}}}$

The density estimates of the circumbinary material of two different works have been considered (Anupama \& Kantharia 2005; Takei et al. 2015). The radius of a "bullet" or knot was taken to vary between $5 \times 10^{14}$ to $1 \times 10^{15} \mathrm{~cm}$ and the density from $1.5 \times 10^{3} \mathrm{~cm}^{-3}$ to $5.5 \times 10^{3} \mathrm{~cm}^{-3}$, following S12 and Verro (2015), see Fig. 14. Density estimates from Takei et al. (2015) lead to hydrodynamic timescales of 333 to $1965 \mathrm{yr}$, whereas those of Anupama \& Kantharia (2005) vary from 1775 to 8239 yr. Using 

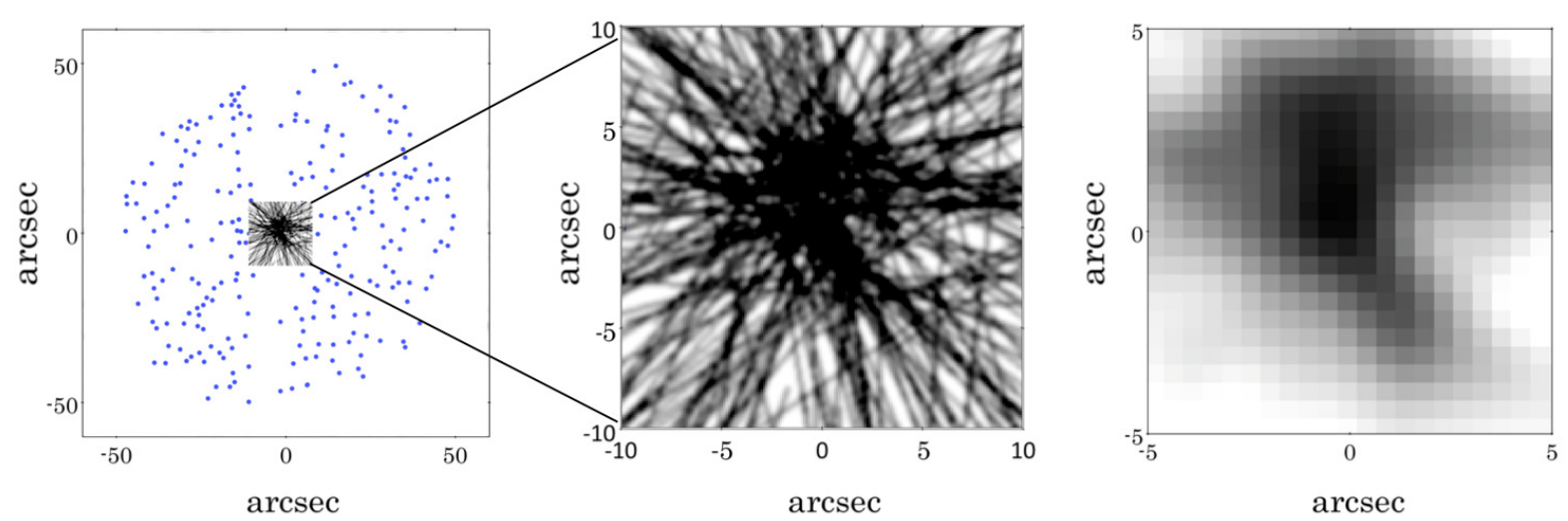

Fig. 11. Criss-cross mapping technique applied to GK Per observations. The criss-cross map shows a radial outflow with a discernible shift $2^{\prime \prime}$ to the north, although within the noise from the observations.

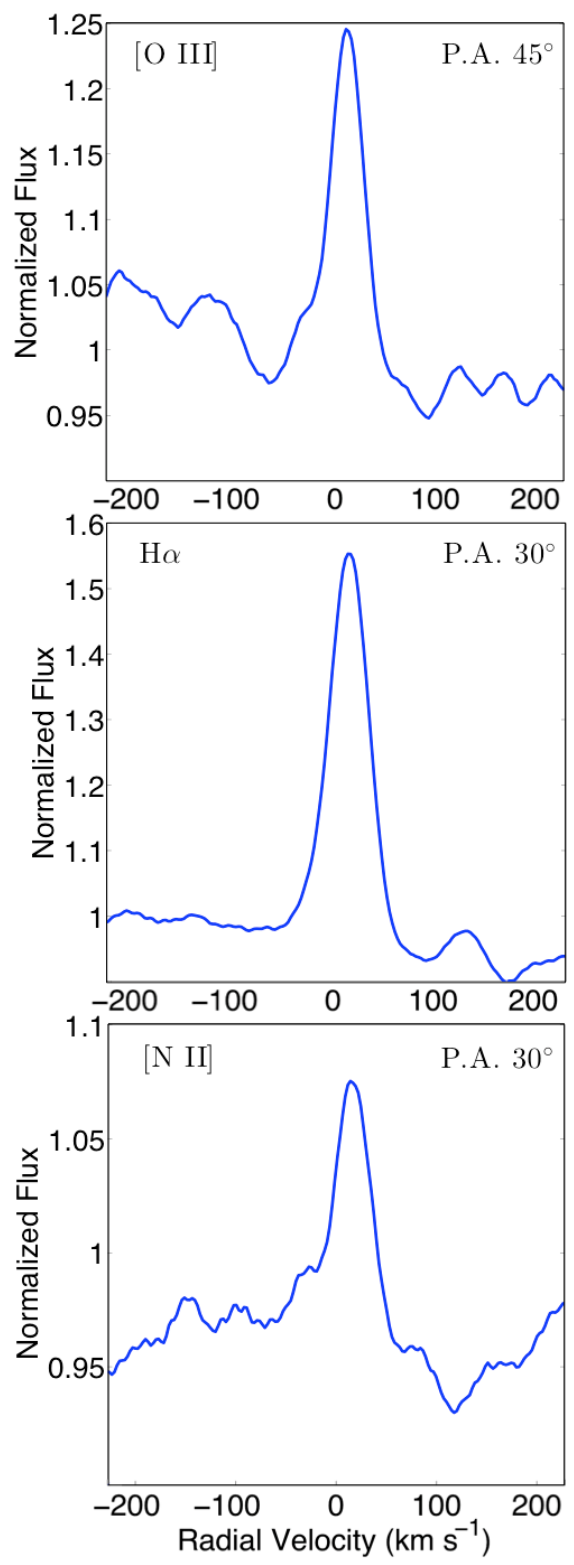

Fig. 12. Top panel: resolved [O III] $5007 \AA$ line of the jet-like feature taken on 2014-Nov-28 at a PA $=30^{\circ}$, as illustrated in Fig. 1. The two lower panels are of the $\mathrm{H} \alpha 6563 \AA$ and [N II] $6583 \AA$ spectral lines from a slit placed at $\mathrm{PA}=45^{\circ}$. These observations are summarised in Table 2a. the densities mentioned above, we can estimate the hydrodynamic timescale according to the velocity half-lives of S12 (58 yr and $220 \mathrm{yr}$ ) and of L12 (100 to $6000 \mathrm{yr}$ ). L12 made their velocity half-life calculation based on a value for the initial expansion velocity of $1340 \mathrm{~km} \mathrm{~s}^{-1}$ from Pottasch (1959) and compared it to their own observations. However, there is a range in the initial expansion velocity values for GK Per from 1240 to $1700 \mathrm{~km} \mathrm{~s}^{-1}$ (Anupama \& Kantharia 2005; and Seaquist et al. 1989, respectively) rendering this type of treatment subject to large uncertainties. Destruction of a knot follows several crushing times (Pittard 2007). Given the uncertainties, it is not possible to determine whether the main body of knots have been slowed or continue to expand more or less freely. However, our analysis suggests that some knots in the polar directions, where the densities are higher, have been slowed enough to significantly shape the nebula. Future monitoring should yield further details of the evolving knot interaction pattern, see bottom panel of Fig. 13.

Over time a knot will undergo several mass changing and shaping effects due to its environment such as photo-evaporation and thermal evaporation. Hydrodynamic ablation can occur via a shock transmitted through a clump; on reaching the end of the clump a strong rarefaction is reflected leading to expansion downstream that is accompanied by a lateral expansion (Pittard 2007). Lateral expansion from hydrodynamic ablation gives values of the order of $14 \%$ of the velocity of a knot away from the GK Per central system, which is caused by the high pressure in the knot versus the lower pressure of the surroundings and should be uniform along the length of the tail. The knot tails may also be experiencing Kelvin-Helmholtz instabilities. However the knots of GK Per are distorted on a longer wavelength and amplitude than would be expected from Kelvin-Helmholtz instability alone.

In a clump-wind interaction their relative velocities must be considered as it is believed to be the main mechanism in the shaping of the clumps. Under more uniform conditions, subsonic clumps have long tails and their supersonic counterparts display short stubby tails (Pittard et al. 2005). There are a variety of tail shapes present in GK Per suggesting diverse local flow conditions. Nevertheless, we suggest that the tail shapes are due to winds external to the clumps, specifically that they are due to dwarf nova winds. Several knots that appear to be experiencing an interaction with a following wind can be identified in the HST images. Five of the best examples were selected that had a sinusoidal tail shape of similar wavelength and amplitude along the outer edge of the shell such that inclination assumptions of individual knots are avoided. These wavy tails can be attributed to shaping by the dwarf nova winds that are thus 

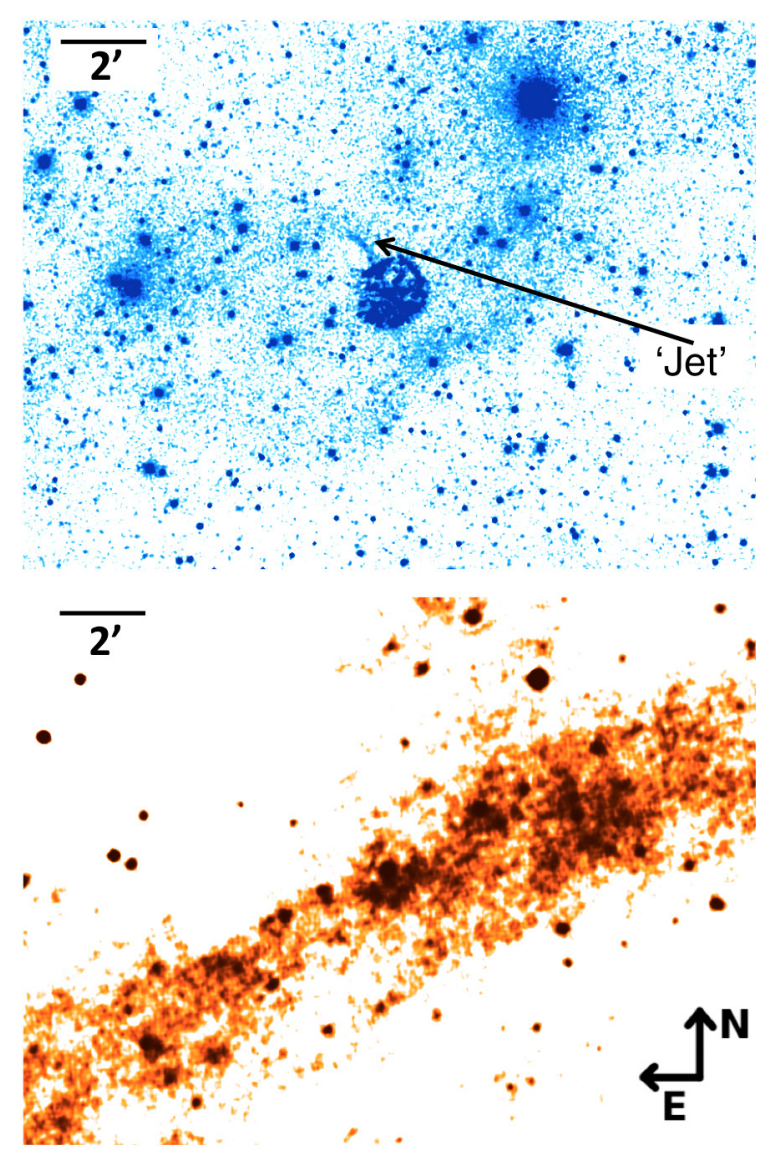

Fig. 13. Top panel: binned and stretched $\mathrm{H} \alpha$ image from the Mayall telescope where the jet-like feature is clearly visible to the NE, while the surrounding planetary nebula can also be seen. Bottom panel: WISE band 3 (resolution $=6.5^{\prime \prime}$ ) illustrates the IR emitting material with which the optical shell is recombining. Strong outflow in the polar directions derived from the model presented here can also be seen.

found to have a velocity of $\sim 4400 \mathrm{~km} \mathrm{~s}^{-1}$. However, it should be noted that the dwarf nova outbursts vary in peak magnitude, meaning different energetics leading to different outburst velocities. Bianchini et al. (1986) gave a velocity estimate of the dwarf nova winds associated with GK Per of a few $1000 \mathrm{~km} \mathrm{~s}^{-1}$. Evidence for these wavy tails can be seen in the P-V arrays in velocity space in Fig. 2, where a sinusoidal change in velocity can be seen while looking down the length of the knot. Simply considering the time to traverse the system the dwarf nova responsible for the shaping of the tails in 1997 would be from $\sim 1969$. A better understanding of the interaction between knots and the dwarf nova winds could be achieved with detailed hydrodynamical simulations equivalent to Pittard et al. (2005, 2009) and Alūzas et al. (2014) where alternative hydrodynamical mechanisms such as the Kelvin-Helmholtz instability could be explained, or even possibly the Richtmyer-Meshkov instability since the dwarf nova episodes may be accelerating ejecta and the clump surfaces are irregular.

The southern bar which appears to be interacting with the shock has been the site of the strongest optical emission ever since the first image of the nebulosity by Barnard (1916). The careful examination of individual knots by L12 gave a mean weighted kinematical age of $118 \pm 12 \mathrm{yr}$ for the nova shell (compared to the shell at $103 \mathrm{yr}$ old in 2004), with no hint of directional dependancies. In contrast to the work carried out by L12, Duerbeck (1987) proposed that the GK Per nova shell was

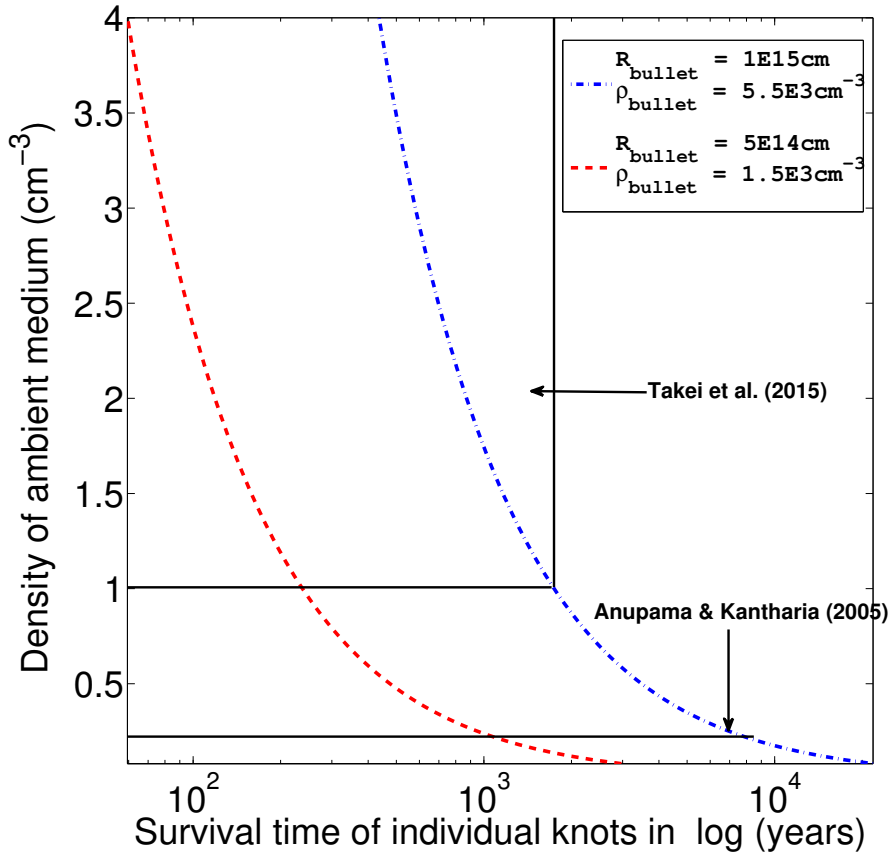

Fig. 14. Bullet crushing time calculations. The blue line (dot-dashed) shows the maximum length of time, depending on density, that an individual clump would remain in motion given observed constraints on the system. The red line (dashed) represents the shortest amount of time. The area of maximum likelihood is the area between the curves.

decelerating by $10.3 \mathrm{~km} \mathrm{~s}^{-1} \mathrm{yr}^{-1}$ and calculated the value for the expansion of the shell at $1200 \mathrm{~km} \mathrm{~s}^{-1}$. The fastest knot found using the formalisms put forward by L12 is $1190 \mathrm{~km} \mathrm{~s}^{-1}$ and the lowest expansion velocity derived is $267 \mathrm{~km} \mathrm{~s}^{-1}$. It is interesting to note that all of the knots that exceed $1000 \mathrm{~km} \mathrm{~s}^{-1}$ (around $7 \%$ of all knots measured) are located on the outer limb. The knots below $600 \mathrm{~km} \mathrm{~s}^{-1}(13 \%)$ all reside inside the nebular boundary. There are only two recorded exceptions to the latter in the NE and whose P-V arrays show them to be interacting, e.g. Fig. 4 top row and Fig. 5 NE knot. The projected appearance of knots along the barrel morphology means some knots will be orientated perpendicular to our line of sight, as we see in the NE, e.g. Fig. 6a, or the corresponding blue side of the shell where lack of emission to the NW can be seen.

An unexpected result here is that the derived morphology has a less extended axial ratio than expected, which goes against the grain of some of the shapes of recently modelled nova shells, e.g. RS Ophiuchi, V2672 Ophiuchi, and KT Eridani (Ribeiro et al. 2009, 2013; Munari et al. 2010). Lloyd et al. (1997) used a 2.5D hydrodynamic code to investigate remnant shaping for a variety of speed classes and produced rings, blobs, and caps as expected, but also created oblate remnants. Later, Porter et al. (1998) included the effects of a rotating accreted envelope; surprisingly, the first panel in their Fig. 2 bares quite a resemblance to the morphology derived for GK Per in this work, and to model A used by Ribeiro et al. (2011). Emission in Band 3 of the WISE data (bottom panel of Fig. 13) suggests a large amount of material in the polar regions, possibly having a significant affect on the velocity of material moving in the polar directions.

\section{Conclusions}

In this work different axisymmetric models were considered in order to put fusther effort towards the understanding of the complex morphology of the old nova shell associated with 
GK Per, where we find a barrel equatorial feature with polar cones to give the best fit. In the light of our new echelle spectra gathered in November 2014, March 2015, and the WISE data archives, a new hypothesis on the origin of the mysterious jetlike feature has been put forward, i.e. that it is part of the ancient planetary nebula.

We propose that the wavy tails of knots in the nova shell may be due to shaping by dwarf nova winds. This allows us to derive dwarf nova wind velocities of about $4400 \mathrm{~km} \mathrm{~s}^{-1}$, although sophisticated hydrodynamical simulations would be needed to test the robustness of this hypothesis. Based on Doppler map profiles of the overall distribution of the nova shell knots it was found that a spherical shell, warped or otherwise, does not adequately explain the red-blue spread found in observations discussed here, although a spherical shell is a good first approximation to the overall shape of the knot distribution. Instead a cylindrical form with an axial ratio close to unity is found to fit best, and also shows a remarkable resemblance to the imaging data. After the application of the cylindrical shape to the main body of the nova shell, polar features were included to account for the large number of knots not explained by the barrel.

The jet-like feature is most likely part of the surrounding planetary nebula owing to its low observed velocity and structure. Following the emission lines in our new observations through the shell, they are enhanced at a lower velocity at the area of interaction as observed in radio and X-ray observations. From the spatial modelling conducted it cannot be said whether the surrounding planetary nebula is indeed bipolar or cylindrical in structure with the polar over-densities (e.g. WISE band 3) attributable to both scenarios. Deep high-resolution echelle spectroscopy is needed to decipher between the two scenarios and to then test the efficiency of the shaping mechanisms.

Acknowledgements. The authors would like to thank the staff at the SPM and Helmos observatories for the excellent support received during observations. The Aristarchos telescope is operated on Helmos Observatory by the Institute for Astronomy, Astrophysics, Space Applications and Remote Sensing of the National Observatory of Athens. Based upon observations carried out at the Observatorio Astronomico Nacional on the Sierra San Pedro Martir (OAN-SPM), Baja California, México. This research uses services or data provided by the NOAO Science Archive. NOAO is operated by the Association of Universities for Research in Astronomy (AURA), Inc., under a cooperative agreement with the National Science Foundation. Based on observations made with the Nordic Optical Telescope, operated by the Nordic Optical Telescope Scientific Association at the Observatorio del Roque de los Muchachos, La Palma, Spain, of the Instituto de Astrofisica de Canarias. The data presented here were obtained in part with ALFOSC, which is provided by the Instituto de Astrofisica de Andalucia (IAA) under a joint agreement with the University of Copenhagen and NOTSA. Some of the data presented in this paper were obtained from the Mikulski Archive for Space Telescopes (MAST). STScI is operated by the Association of Universities for Research in Astronomy, Inc., under NASA contract NAS526555. Support for MAST for non-HST data is provided by the NASA Office of Space Science via grant NNX09AF08G and by other grants and contracts. This publication makes use of data products from the Wide-field Infrared Survey Explorer, which is a joint project of the University of California, Los Angeles, and the Jet Propulsion Laboratory/California Institute of Technology, funded by the National Aeronautics and Space Administration. E. Harvey wishes to acknowledge the support of the Irish Research Council for providing funding for this project under their postgraduate research scheme. S.A. gratefully acknowledges a postdoctoral fellowship from the Brazilian Agency CAPES (under their program: "Young Talents Attraction" - Science Without Borders; A035/2013). The authors greatly benefitted from discussions with W. Steffen, J. Meaburn, M. Lloyd, T. Jurkic, C. Neilson, and V.A.R.M. Ribeiro. We also wish to thank the anonymous referee for helpful and thoughtful comments and suggestions.

\section{References}

Akras, S., \& Steffen, W. 2012, MNRAS, 423, 925

Alūzas, R., Pittard, J. M., Falle, S., \& Hartquist, T. W. 2014, MNRAS, 444, 971 Anupama, G. C., \& Kantharia, N. G. 2005, A\&A, 435, 167
Anupama, G. C., \& Prabhu, T. P. 1993, MNRAS, 263, 335

Balick, B. 1994, ApSS, 216, 13

Balman, Ş. 2005, ApJ, 627, 933

Barnard, F. 1916, Harvard College Observatory Bulletin, 1

Bianchini, A., Sabbadin, F., \& Hamzaoglu, E. 1982, A\&A, 106, 176

Bianchini, A., Sabbadin, F., Favero, G. C., \& Dalmeri, I. 1986, A\&A, 160, 367

Bode, M. F., \& Evans, A. 2008, Classical Novae, 2nd edn. (University Press)

Bode, M. F., Seaquist, E. R., Frail, D. A., et al. 1987, Nature, 329, 519

Bode, M. F., O’Brien, T. J., \& Simpson, M. 2004, ApJ, 600, L63

Casanova, J., Jose, J., Garcia-Berro, E., Calder, A., \& Shore, S. N. 2011, A\&A, 527, A5

Clyne, N., Akras, S., Steffen, W., et al. 2015, A\&A, 582, A19

Cordova, F. A., \& Mason, K. O. 1982, ApJ, 260, 716

Couderc, P. 1939, Annales d'Astrophysique, 2, 271

Crampton, D., Fisher, W. A., \& Cowley, A. P. 1986, ApJ, 300, 788

Duerbeck, H. W. 1987, ApSS, 131, 461

Duerbeck, H., \& Seitter, W. 1987, ApSS, 131, 467

Eaton, J. W., Bateman, D., Hauberg, S., \& Wehbring, R. 2014, GNU Octave version 3.8.1 manual: a high-level interactive language for numerical computations (CreateSpace Independent Publishing Platform)

Ederoclite, A. 2005, Ph.D. Thesis, Trieste University, Astronomy Department

Evans, A., Gehrz, R. D., Woodward, C. E., \& Helton, L. A. 2014, MNRAS, 444, 1683

Harrison, T. E., Bornak, J., McArthur, B. E., \& Benedict, G. F. 2013, ApJ, 767, 7

Harrison, T. E., \& Hamilton, R. T. 2015, AJ, 150, 19

Helton, L., Evans, A., Woodward, C., \& Gehrz, R. 2011, EAS Pub, 46, 407

Hillman, Y., Prialnik, D., Kovetz, A., \& Shara, M. M.2016, ApJ, 819, 168

Kafka, S., \& Honeycutt, R. K. 2004, AJ, 128, 2420

Kastner, J., \& Gatley, I. 2000, ASP Conf Proc, APN II, 199, 355

Kraft, R. P. 1964, ApJ, 139, 457

Lawrence, S., MacAlpine, G., Uomoto, A., et al. 1995, AJ, 109, 2635

Liimets, T., Corradi, R. L. M., Santander-García, M., et al. 2012, ApJ, 761, 34

Lloyd, H. M., O’Brien, T. J., \& Bode, M. F. 1997, MNRAS, 284, 137

Mauche, C. W., \& Raymond, J. C. 1987, ApJ, 323, 690

McLaughlin, D. 1960, Stellar Atmospheres, Vol. VI (Chicago University Press)

Meaburn, J., Lopez, J., Gutierrez, L., et al. 2003, Rev. Mex. Astron. Astrofis., 39, 185

Morales-Rueda, L., Still, M. D., Roche, P., Wood, J. H., \& Lockley, J. J. 2002, MNRAS, 329, 597

Munari, U., Ribeiro, V., Bode, M., \& Saguner, T. 2010, MNRAS, 410, 525

Nordhaus, J., \& Blackman, E. G. 2006, MNRAS, 370, 2004

Osaki, Y. 1996, PASP, 108, 39

Pittard, J. M. 2007, Astrophys. Space Sci. Proc., 245

Pittard, J. M., Dyson, J. E., Falle, S. A. E. G., \& Hartquist, T. W. 2005, MNRAS, 361, 1077

Pittard, J. M., Falle, S. A. E. G., Hartquist, T. W., \& Dyson, J. E. 2009, MNRAS, 394, 1351

Poludnenko, A. Y., Frank, A., \& Mitran, S. 2004, ASP Conf. Proc., APN III, 313, 434

Porter, J. M., O’Brien, T. J., \& Bode, M. F. 1998, MNRAS, 296, 943

Pottasch, S. 1959, Annales d'Astrophysique, 22, 297

Redman, M. P., Meaburn, J., \& Holloway, A. J. 2002, MNRAS, 332, 754

Ribeiro, V., Bode, M. F., Darnley, M. J., et al. 2009, ApJ, 703, 1955

Ribeiro, V. A. R. M., Darnley, M. J., Bode, M. F., et al. 2011, MNRAS, 412

Ribeiro, V., Bode, M. F., Darnley, M. J., et al. 2013, MNRAS, 433, 1991

Roy, N., Kantharia, N. G., Eyres, S. P. S., et al. 2012, MNRAS, 427, L55

Sabbadin, F., \& Bianchini, A. 1983, A\&AS, 54, 393

Schaefer, G. H., Brummelaar, T., Gies, D. R., et al. 2014, Nature, 515, 234

Scott, A. D., Rawlings, J. M. C., \& Evans, A. 1994, MNRAS, 269, 707

Seaquist, E., Bode, M., Frail, D., et al. 1989, ApJ, 344, 805

Shara, M. 1981, ApJ, 243, 926

Shara, M. M., Zurek, D., Marco, O. D., et al. 2012, AJ, 143, 149

Slavin, A., Brien, T. O., \& Dunlop, J. 1995, MNRAS, 276, 353

Steffen, W., \& Koning, N. 2011, AJ, 141, 76

Steffen, W., Koning, N., Wenger, S., Morisset, C., \& Magnor, M. 2011, IEEE

Transactions on Visualization and Computer Graphics, 17, 454

Takei, D., Drake, J. J., Yamaguchi, H., et al. 2015, ApJ, 801, 92

Townsley, D. M., \& Bildsten, L. 2005, ApJ, 628, 395

Tweedy, R. W. 1995, ApJ, 438, 917

Verro, K. 2015, Physical Properties of the Nova Remnant Nova Persei 1901

Warner, B. 1995, Cataclysmic Variable stars, 1st edn. (Cambridge University Press), Vol. 1

Watson, M. G., King, A. R., \& Osborne, J. 1985, MNRAS, 212, 917

Wesson, R., Barlow, M. J., Corradi, R. L. M., et al. 2008, ApJ, 688, L21 


\section{Appendix A: Data from new observations presented}

\section{in this work}

Table A.1. $x$ and $y$ positions of knots normalised to 2007 NOT data epoch for easier comparison with L12.

\begin{tabular}{|c|c|c|c|}
\hline $\begin{array}{l}x \\
\operatorname{arcsec}\end{array}$ & $\begin{array}{l}y \\
\operatorname{arcsec}\end{array}$ & $\begin{array}{l}\text { Radial velocity } \\
\left(\mathrm{km} \mathrm{s}^{-1}\right)\end{array}$ & Feature \\
\hline-32.8 & 29.0 & 137 & barrel \\
\hline-31.8 & 23.8 & 450 & barrel \\
\hline-30.5 & 32.5 & -20 & barrel \\
\hline-30.5 & 26.5 & -29 & barrel \\
\hline-30.3 & 20.6 & 465 & barrel \\
\hline-30.3 & 15.5 & 658 & barrel \\
\hline-28.5 & 4.0 & 658 & barrel \\
\hline-27.4 & -3.9 & 888 & barrel \\
\hline-24.9 & -15.2 & -561 & pole \\
\hline-24.0 & -21.3 & 574 & barrel \\
\hline-23.2 & -24.4 & -523 & pole \\
\hline-22.7 & -13.8 & -768 & pole \\
\hline-21.3 & -34.5 & 485 & barrel \\
\hline-20.8 & 35.6 & -55 & barrel \\
\hline-19.6 & -35.9 & -440 & barrel \\
\hline-19.1 & -33.2 & 528 & barrel \\
\hline-18.8 & -36.5 & -653 & pole \\
\hline-16.1 & -47.0 & -113 & barrel \\
\hline-10.0 & -18.3 & -690 & barrel \\
\hline-7.8 & 12.8 & -532 & barrel \\
\hline-7.0 & -12.9 & -110 & barrel \\
\hline-3.7 & -6.7 & 64 & barrel \\
\hline-2.7 & 47.8 & -108 & barrel \\
\hline-0.3 & 46.2 & 64 & barrel \\
\hline 13.6 & 34.4 & -29 & barrel \\
\hline 15.8 & 31.3 & 392 & pole \\
\hline 19.0 & 29.6 & 580 & pole \\
\hline 30.9 & 17.5 & 653 & pole \\
\hline 35.2 & 12.8 & -729 & barrel \\
\hline 39.2 & 8.0 & 498 & pole \\
\hline 40.6 & 6.0 & 290 & pole \\
\hline 44.1 & 3.5 & -222 & barrel \\
\hline 47.4 & 0.1 & -273 & barrel \\
\hline
\end{tabular}

Notes. The positional matching errors are discussed at the end of Sect. 2.1 and the positional matching of knots between the two WCS matched data sets was done by matching knot shapes and centroids with consideration of their expected motion in the plane of the sky. Given that additional positional matching errors may arise from flux variations along a single knot between the two epochs (see S12, Fig. 10) or misidentification of a knot, average errors are of the order of $\pm 1^{\prime \prime}$ and maximum errors are expected to be up to $3^{\prime \prime}$. The errors in the radial velocity are of the order of $\pm 22 \mathrm{~km} \mathrm{~s}^{-1}$. As the MES wavelength range does not fully cover the three [N II] $6548 \AA$, H $\alpha 6563 \AA$, and [N II] $6583 \AA$ emission lines, the following measurements were done with the [N II] $6583 \AA$ where possible, and the [N II] 6548 A elsewhere; the $\mathrm{H} \alpha 6563 \AA$ line velocities were also measured as a sanity check. 